\title{
Career pathways, part 3
}

\author{
In this instalment of Career pathways, Jing Fan and Edward A. Phelps reflect on fostering their newly formed \\ research programs in the face of challenges both familiar and new.
}

\author{
Jing Fan and Edward A. Phelps
}

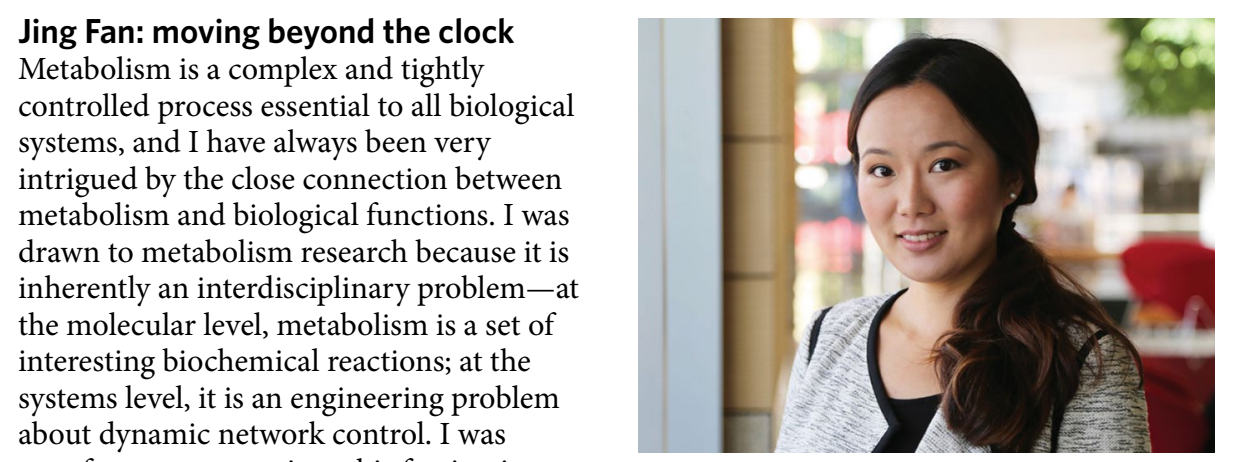
very fortunate to get into this fascinating field while the 'renaissance' of metabolism research was starting. I did my $\mathrm{PhD}$ studies in the laboratory of Josh Rabinowitz at Princeton University, and was trained in some of the cutting-edge approaches that fuelled this renaissance. I enjoyed the opportunity to use my chemistry and quantitative background to make discoveries in biological problems. It was a really great and inspiring training experience that set the foundation for my career.

I started my own lab at the Morgridge Institute for Research and the University of Wisconsin-Madison in 2017. The article we published in Nature Metabolism (G. L. Seim et al. Nat. Metab. 1, 731-742; 2019) not only is the first major research article from my lab but also represents a new direction for me. Shortly after I started my independent research program, I found myself devoting major efforts to understanding the metabolism of immune cells-a cell type I had never worked with during my training. I was deeply intrigued by the plasticity of these cells. Macrophages can respond to various environmental cues and perform a range of functions, from killing to healing. What about their metabolism during this dynamic process? As we delved into this new area, my team and I were constantly stimulated by new discoveries, ideas and questions. But sometimes, particularly when I looked around and saw many young scientists launch successful careers directly building on their postdoctoral work, I wondered whether my path was a risky one. I worried about whether grant reviewers would rather see something I already had a record for; whether I was
Credit: Morgridge Institute for Research

'falling behind' by building a major project in my brand-new lab 'from scratch'; and whether I could find good students and postdocs willing to take this path with me. In the end, my scientific passion for understanding the fundamental principles that underpin dynamic metabolic regulation and the interplay between metabolism and cell function just led me deeper into the realm of immunometabolism, where I saw many interesting open questions and opportunities. I knew that, although I was getting into a new biological system, at the core of it were the same types of metabolism problems that I was equipped to engage with, analyse and understand. I knew I could make a contribution to this field; I just needed a milestone, such as a publication, to demonstrate what we could do-the article we published in Nature Metabolism was that milestone.

This article set the foundation for many of the current studies in my lab. It exemplifies the set of questions that we are interested in answering and are able to address: What are the important metabolic transitions during an immune response (or other dynamic physiological transitions)? How do they happen mechanistically? Why is such metabolic reprogramming important for cells? We have made much progress since the publication of our first paper. Some of our projects zoom in on the key enzymes identified in the previous paper, looking deeper at the molecular level to investigate their regulatory mechanisms. Some projects go further in understanding how the identified metabolic rewiring orchestrates immune function. Other projects are more 'in parallel'-looking at similar questions but focusing on different pathways, in different innate immune cells during different immune responses. With more discoveries come more questions. This keeps us motivated on this exciting journey.

I am very grateful to have had great support on this journey. A value at my institution that I embrace is to pursue 'fearless science'- to do our best to generate real meaningful knowledge instead of overfocusing on small-minded merits. Last year, when I got feedback from our scientific advisory board, I was very encouraged to see that it recognized my most impressive progress as "carving out an entirely new set of discoveries and a new direction for her lab in the field of immunometabolism." I also deeply appreciate the kind support from many former and current mentors and colleagues. And I have learned a lot from my collaborations with experts in immunology and other areas. What means the most to me is my lab. I am very proud of my team of talented students, who are always motivated to learn new things and work hard together to solve problems. Their excellent work, particularly that by Gretchen Seim, the first author of our Nature Metabolism paper, has been essential for launching this exciting new direction. It is truly rewarding as a PI to work with and develop alongside my trainees.

Starting a lab and figuring out a new path requires a good balance. We need to pursue the most exciting and important scientific questions, while responsibly using start-up resources to ensure the success of our students and the continued growth of the lab. Everyone's path is different. I have seen successful examples across a spectrumsome scientists build their new labs upon projects from their postdoc and gain momentum quickly, whereas others pioneer new areas distinct from their previous work. The reality is that, regardless of the direction of our labs starting out, as young scientists, we are often put against a 'clock'. 
For instance, the standard for the tenure process is essentially calibrated according to 'normal' productivity within the first few years, and early-career grants are to some extent evaluated on the basis of productivity in the first years. As a result, some stress about the timeframe is almost inevitable. But, at the end of the day, I chose my path not according to a clock of 3 years or 5 years, but with a view toward a long career of meaningful, passionate research. I remember that when I was first recruited to my current position, I had a great conversation with my director, Dave Pagliarini, and the institute leader, Brad Schwartz. They told me that the expectation is not to just 'check off the boxes in the tenure package' but to do research that has real impact. In that moment, I knew I was in the right place.

I think science is becoming increasingly interdisciplinary. This is exactly why metabolism is so attractive to me in the first place-it is both ubiquitous and unique. All cells need metabolism, and metabolic processes are governed by some fundamental principles, yet, in different biological contexts, metabolism can be quite distinct, and it comes with unique sets of questions. As such, the field of metabolism offers fertile ground for us as researchers to both delve deeper into the principles and expand more broadly into new biological problems. I keep reminding myself and my lab of the big picture and why we want to be scientists, particularly at times when the worry that working outside our specialty might make us fall behind the clock gets in the way of discoveries. The nature of scientific research is building on what we already know to study what we do not yet know. So we should cherish the privilege of chasing after our own questions, keep finding new frontiers, and grow from continued learning and creating.

\section{Edward A. Phelps: it starts with mentorship}

Around the middle of my postdoctoral tenure, I experienced a 'eureka' moment. I remember it clearly: I arrived at the microscopy core at the École Polytechnique Fédérale de Lausanne (EPFL) in Switzerland one chilly and overcast January afternoon with freshly prepared microscope slides. The imaging facility was busy with other researchers silently toiling away at their scopes. My eyes were still adjusting to the dim lighting as I booted up the confocal. It had been 3 years since I'd uprooted my entire existence in Atlanta, Georgia, to pursue a once-in-a-lifetime opportunity for an international postdoctoral fellowship in Switzerland. My recent months at the bench had been marked by characterizing the

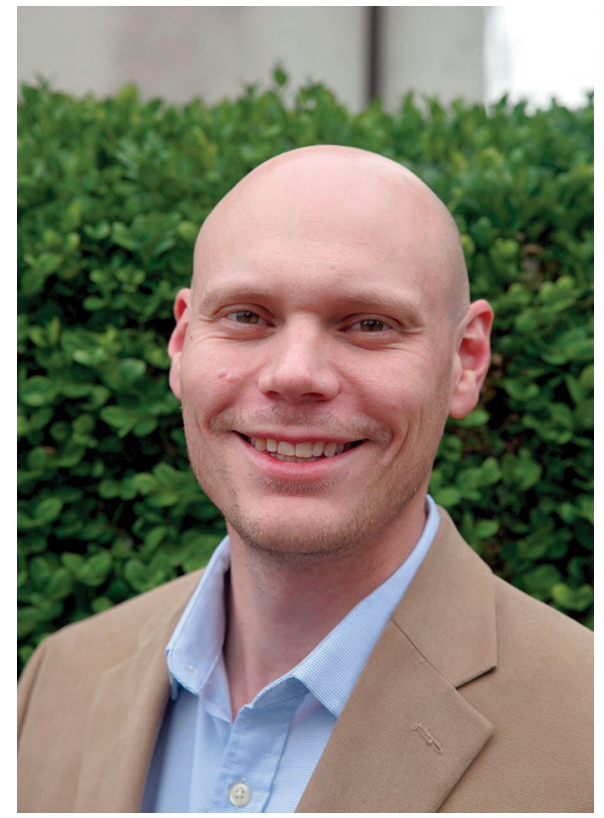

Credit: Amanda Phelps

subcellular distribution of a protein called glutamic acid decarboxylase 65 (GAD65) found in pancreatic beta cells.

Before starting the project, I had never heard of GAD65, but at that time, my life revolved around its rhythms. After many dead ends and false starts, I was beginning to feel my world unravelling-the pressure to publish and obtain an independent assistant-professor position haunted me. My wife and I had just had our first child, and she had delayed her career as an attorney to support my scientific journey abroad. Although I desperately wanted to succeed, my research project was not going well. My experiments were progressing into strange territory, in which the results inexplicably, yet consistently, contradicted accepted biology.

On that particular day, I tried something different for the first time. Instead of my standard approach, I decided to examine the enzymatic product of GAD65, GABA, a neurotransmitter also found in pancreatic beta cells. Expecting another round of indecipherable puncta scattered around the cytoplasm, I started up the microscope and brought the cells slowly into focus. Peering through the eyepiece, I stopped dead in my tracks. The GABA was not in punctate vesicles, as is typical of GABA-producing cells; it was everywhere-uniformly distributed throughout the cytosol of the beta cells. Involuntarily, I jumped up from my seat and yelled out, "Yes!" and then quickly apologized to the startled researcher at the workstation next to me for my very
un-Swiss outburst. In that moment, I made the first step in the long journey towards solving a 30 -year-old mystery in the islet GABA system.

It took 5 more years of effort after that first microscope slide to finally publish our results in Nature Metabolism on the role of GABA in pancreatic islets (D. Menegaz et al. Nat. Metab. 1, 1110-1126; 2019). Although it was exciting that this story was the first publication from my independent laboratory, its publication highlights the beauty of collaboration, being the culmination of coequal collaborations among my lab at the University of Florida (UF), Steinunn Baekkeskov's lab at EPFL in Switzerland and Alejandro Caicedo's lab at the University of Miami.

I opened the doors to the Phelps laboratory at UF in 2017. Although I followed a mostly conventional academic career path to get to that point, much of that trajectory was also a matter of circumstance. I was accepted into only one university after high school, and for a long time I struggled with the feeling that I did not belong there. After being encouraged by a graduate teaching assistant, I joined a research laboratory as an undergraduate, which turned out to be an awakening for me. I realize now how important that experience was and how the opportunity changed the course of my professional career. After transitioning to the graduate doctoral program at Georgia Tech, I further benefitted from excellent mentors, such as my $\mathrm{PhD}$ advisor, Andrés García, who helped me discover and unlock my passion and drive for science. As a postdoc at EFPL in Switzerland, the opportunity to work and study internationally with support from the Whitaker Foundation (and later from JDRF) was a formative experience professionally and personally. Even as a white immigrant in a predominantly white European country, I experienced firsthand a tiny bit of what it is like to be an immigrant scientist, and struggling to get my bearings in a newly adopted country highlighted for me the importance of empathy for those who leave their homes to pursue their dreams.

Starting my own lab as a tenure-track assistant professor at UF gave me a fresh start. While I had many potential research threads to pursue, I was still passionate about uncovering the nuances of the GABA story, so I took a risk, investing heavily in further developing our findings. I was fortunate to have recruited a talented and intuitive doctoral student, Walker Hagan, who delved into this project, methodically working to uncover the molecular mechanism of GABA release from the cytosol of beta cells. We learned on the 
fly and built the lab without the safety nets present in more senior, established labs, such as seasoned technicians and established protocols. However, I greatly benefited from the mentorship and help of others who contributed essential pieces to this GABA puzzle, such as Steinunn Baekkeskov and Alejandro Caicedo, whose advice was a welcome beacon as we organized our results into a research narrative. It was a big moment for our team when Nature Metabolism agreed to send out the initial manuscript for peer review. Because our manuscript challenged a primary aspect of the accepted model of the islet GABA system, I was concerned about encountering resistance. Despite my fears, the peer reviewers were fair and supportive. My young lab grew emotionally and intellectually, and we expanded our technical capabilities through the support of senior mentors, the peer review process and the editors who guided us through the revisions.

In the time since first opening my lab at UF, we have celebrated victories: building our team, the acceptance of our first paper and being awarded our first grant. And we have also weathered setbacks: untimely rejections, failed experiments and broken equipment. We have survived more than one major hurricane bearing down on the Floridian peninsula. After initially halting all benchwork activities, we are now operating at reduced capacity in the throes of a global pandemic. I myself contracted COVID-19 in March of 2020, experiencing the full litany of symptoms, including respiratory distress, and was lucky to turn the corner before needing hospitalization. Many months later, I am still fighting to fully recover my health. Before the pandemic, getting our first publication in Nature Metabolism helped us build momentum, so it has been difficult to hit the pause button on our progress. Concerns over the impact of the pandemic on both my career path as an early-stage investigator and on the future careers of my amazing graduate students are a heavy weight. Neither of my children were in existence when I started out on my GABA project, and my eldest was in kindergarten by the time it was finally published. All of those sleepless nights, diaper changes, first steps, first words and first bike rides happened alongside the science. During quarantine, my wife, Amanda, and I have done our best to parent, teach and play with our two young daughters, ages 6 and 4, who were out of school for several months, but, like many investigators early in their careers, I worry about the effects that the pressure to keep my head above water in my career will have on myself and my family in this new world of COVID-19.

The world is changing, and while COVID-19 has brought new challenges, it has also exacerbated and brought to light many of the difficulties and inequalities that have long been part of science.

Adapting now to this strange world of social distancing has had its own challenges. Although I feel isolated from my support network, I have no illusions that this time must be even harder for many of my colleagues. My wife made the difficult decision to delay her professional progress so that I could pursue science, something for which I am grateful. The productivity and well-being of scientists who are parents and caregivers, particularly female scientists, is being affected by the circumstances of the pandemic, making caregivers vulnerable to loss of opportunity during these times. Institutions, professional societies and funding agencies should enact policies that are supportive and understanding of the current demands on scientist caregivers to directly respond to this ongoing problem. During COVID-19, we have once again been reminded of the enduring racial inequality in science, in the United States and worldwide. I urge my white colleagues to listen to and support the Black and underrepresented scientists in their departments, to acknowledge their struggles, and to make concrete steps toward greater inclusion and empowerment of historically excluded groups.
Although the world we are living in now can appear more isolating, the example set by my mentors, such as Andrés García, Steinunn Baekkeskov, Alejandro Caicedo and Cherie Stabler, showed me that mentorship is about building up trainees and lighting a path for them to follow. Senior mentors taking a moment to check in on their current and former trainees can make a world of difference for early-stage investigators struggling to find their feet. I love this quote by Oprah Winfrey: "A mentor is someone who allows you to see the hope inside yourself." In my case, during a period in which I have struggled to obtain the funding to continue my GABA research, that hope came from a timely phone call from a mentor. The conversation led to a suggestion for a new direction on where to send my grant proposal, which recently fared very well in review and it is now under consideration for funding-potentially my lab's first major funding award. We are all in need of such support in these trying times, and small acts can make a big difference. Building and investing in a team of individuals with different lived experiences will help us to support one another and drive the creativity that is critical to a thriving lab. I am now learning to set an example for my trainees who will be our lab's legacy. Investing in young scientists from all walks of life and seeing them succeed is my greatest joy in this profession.

\section{Jing Fan (D) 1,2® and Edward A. Phelps (ID)} ${ }^{1}$ Morgridge Institute for Research, Madison, WI, USA. ${ }^{2}$ Department of Nutritional Sciences, University of Wisconsin-Madison, Madison, WI, USA.

${ }^{3}$ J. Crayton Pruitt Family Department of Biomedical Engineering, University of Florida, Gainesville, FL, USA.

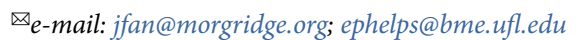

Published online: 4 January 2021

https://doi.org/10.1038/s42255-020-00302-6

Competing interests

The authors declare no competing interests. 